\title{
Produtividade do algodoeiro adensado em segunda safra em resposta à adubação nitrogenada e potássica ${ }^{1}$
}

\author{
Ana Luiza Dias Coelho Borin²*, Alexandre Cunha de Barcellos Ferreira ${ }^{2}$, Valdinei Sofiatti ${ }^{3}$, \\ Maria da Conceição Santana Carvalho ${ }^{4}$, Michelle Christine Gomes Moraes ${ }^{5}$
}

10.1590/0034-737X201764060009

\begin{abstract}
RESUMO
O nitrogênio e o potássio são os nutrientes mais requeridos pelo algodoeiro. O algodão, cultivado em segunda safra, após a colheita da soja, tem sido uma opção dentro dos sistemas de produção do cerrado brasileiro. Este trabalho objetivou avaliar o efeito das adubações nitrogenada e potássica sobre o estado nutricional, os componentes de produção e a produtividade do algodoeiro, cultivado em sistema adensado, semeado em segunda safra. O experimento foi conduzido após a colheita da soja e em delineamento de blocos ao acaso com quatro repetições. Os tratamentos foram distribuídos em esquema fatorial $4 \mathrm{x} 4$, com quatro repetições. $\mathrm{O}$ fator A foram quatro doses de nitrogênio $(0,50$, 100 e $\left.200 \mathrm{~kg} \mathrm{ha}^{-1}\right)$ e o fator B foram quatro doses de $\mathrm{K}_{2} \mathrm{O}\left(0,40,80\right.$ e $\left.160 \mathrm{~kg} \mathrm{ha}^{-1}\right)$, aplicadas em cobertura. Os teores foliares de nitrogênio e de magnésio aumentaram linearmente com as doses de $\mathrm{N}$, enquanto os teores de enxofre e ferro diminuíram (efeito quadrático). As doses de $\mathrm{K}_{2} \mathrm{O}$ resultaram em efeitos positivo e negativo sobre os teores de $\mathrm{K}$ e de $\mathrm{Mg}$, respectivamente. A adubação nitrogenada promove resposta em produtividade de fibra maior do que a adubação potássica, sendo que a taxa de incremento de produtividade de fibra por quilo de nitrogênio foi o dobro da taxa de incremento por quilo de $\mathrm{K}_{2} \mathrm{O}$. As maiores produtividades de fibra foram alcançadas com $148 \mathrm{~kg} \mathrm{ha}^{-1} \mathrm{e} 107 \mathrm{~kg} \mathrm{ha}^{-1} \mathrm{de} \mathrm{N}$ e $\mathrm{K}_{2} \mathrm{O}$, respectivamente.
\end{abstract}

Palavras-chave: nitrogênio; potássio; safrinha; Gossypium hirsutum L.

\section{ABSTRACT \\ Yield of cotton as a second crop in narrow planting in response to nitrogen and potassium fertilization}

Nitrogen and potassium are the most required nutrients for cotton crop. Cotton grown as a second crop, after soybean harvesting, has been an option for crop system in the Brazilian savanna. This study aimed to evaluate the effect of $\mathrm{N}$ and $\mathrm{K}$ fertilization on the nutritional status and the yield components of cotton grown as a second crop, after soybean, and in a narrow planting system. The experiment was carried out adopting a completely randomized block experimental design. The treatments were set up in a $4 \times 4$ factorial arrangement with four replications. Factor A was the four $\mathrm{N}$ doses $\left(0,50,100\right.$, and $\left.200 \mathrm{~kg} \mathrm{ha}^{-1}\right)$ and factor $\mathrm{B}$ was four $\mathrm{K}_{2} \mathrm{O}$ doses $\left(0,40,80\right.$, and $\left.160 \mathrm{~kg} \mathrm{ha}^{-1}\right)$, applied as side-dressing. The $\mathrm{N}$ and $\mathrm{Mg}$ concentration levels in the leaf increased linearly with the $\mathrm{N}$ doses, while the concentration levels of $\mathrm{S}$ and Fe decreased (quadratic effect). The $\mathrm{K}_{2} \mathrm{O}$ doses resulted in positive and negative effects on $\mathrm{K}$ and $\mathrm{Mg}$, respectively, in the leaf. The $\mathrm{N}$ fertilization promoted higher lint yield response when compared with $\mathrm{K}$ fertilization, as lint yield rate per $\mathrm{kg}$ of $\mathrm{N}$ was twice the yield rate per $\mathrm{kg}$ of $\mathrm{K}_{2} \mathrm{O}$. The highest lint yields were reached with $148 \mathrm{~kg} \mathrm{ha}^{-1}$ and $107 \mathrm{~kg} \mathrm{ha}^{-1}$ of $\mathrm{N}$ and $\mathrm{K}_{2} \mathrm{O}$, respectively.

Key words: nitrogen; potassium; late season; Gossypium hirsutum L.

Submetido em 15/05/2015 e aprovado em 30/10/2017

${ }^{1}$ Trabalho financiado pelo Fundo de Incentivo à Cultura do Algodão em Goiás - Fialgo.

Embrapa Algodão - Núcleo Cerrado, Santo Antônio de Goiás, Goiás, Brasil. ana.borin@embrapa.br; alexandre-cunha.ferreira@embrapa.br

${ }^{3}$ Embrapa Algodão, Campina Grande, Paraíba, Brasil. valdinei.soffiati@embrapa.br

${ }^{4}$ Embrapa Arroz e Feijão, Santo Antônio de Goiás, Goiás, Brasil. maria.carvalho@embrapa.br

${ }^{5}$ Universidade Federal de Goiás, Campus Samambaia, Goiânia, Goiás, Brasil. michelle_cgdm@ @otmail.com

*Autora correspondente: ana.borin@embrapa.br 


\section{INTRODUÇÃO}

A região do Cerrado concentra $94 \%$ da produção brasileira de algodão (Conab 2014), sendo cada vez mais comum o cultivo em segunda safra, normalmente após a colheita da soja precoce, ou, em menor escala, após a do feijão. No Mato Grosso, maior estado produtor, na safra de 2013/2014, aproximadamente $70 \%$ do algodão foi semeado em segunda safra (Galbieri et al. 2014), comumente conhecida como safrinha. A intenção inicial dessa alteração da época de semeadura era a redução de custo, uma vez que o ciclo reduzido implicaria menor número de aplicações de pesticidas. Com essa mudança da época de semeadura, diminuíram-se a disponibilidade de água, o crescimento das plantas e o potencial produtivo do algodoeiro, portanto, acredita-se que ocorram alterações na demanda de nutrientes. Outra mudança no sistema de produção, que tem sido adotada em algumas áreas que cultivam em segunda safra, foi a redução do espaçamento entre linhas, de $0,76 \mathrm{~m}$ para $0,45 \mathrm{~m}$, objetivando melhorar a distribuição de plantas por área e, consequentemente, o controle de plantas daninhas e a eficiência no uso de água, luz e nutrientes. $\mathrm{O}$ adensamento antecipa o pico de absorção de nutrientes (Rosolem et al. 2012), sendo que a máxima demanda por nitrogênio é atingida, precocemente, em espaçamentos menores (Hutmacher et al. 2004), sugerindo aplicações antecipadas de nitrogênio e de potássio em cobertura (Rosolem et al. 2012).

Dentre os nutrientes, o nitrogênio $(\mathrm{N})$ e o potássio $(\mathrm{K})$ são os mais requeridos pelo algodoeiro, e a forma mais, comumente, utilizada para fornecimento de ambos é a aplicação mineral via fertilizantes. As recomendações de $\mathrm{N}$ para a máxima eficiência econômica em algodoeiro, nas condições brasileiras, estão na faixa de $120 \mathrm{~kg} \mathrm{ha}^{-1}$ a 175 $\mathrm{kg} \mathrm{ha}^{-1}$ e variam em função da cultura anterior, do teor de matéria orgânica e da produtividade almejada. A aplicação da dose adequada de $\mathrm{N}$ evita o desperdício de fertilizante, a perda de produtividade de fibra (Rinehardt et al. 2004), o crescimento vegetativo exagerado do algodoeiro, o atraso na colheita, o aumento da pressão de pragas e a possibilidade de contaminação ambiental (Hutmacher et al. 2004). O aumento do custo de fertilizantes nitrogenados, associado às perdas e suas consequências ambientais têm pressionado a busca por maior eficiência de uso de $\mathrm{N}$ nos sistemas de produção de algodão (Rochester et al. 2007).

O potássio tem importância vital para o crescimento e o metabolismo das plantas sendo ativador de mais de 60 reações enzimáticas. Além disso, mantém o potencial osmótico e a absorção de água durante o desenvolvimento da fibra; sua deficiência reduz a produtividade e a qualidade de fibra (Oosterhuis, 2001). O algodão semeado em segunda safra, com menor disponibilidade de água, pode apresentar mudanças significativas com relação à absorção de potássio. Isto ocorre porque a maior parte do potássio é transportada até a raiz por difusão, processo altamente dependente do teor de água do solo (Oliveira et al. 2004) e, quando a umidade do solo diminui, o nutriente não é difundido, podendo ocasionar deficiências.

O cultivo adensado do algodoeiro tem sido uma opção de segunda safra, em sucessão a soja. Nessa condição, há maior limitação quanto a disponibilidade de água e temperaturas mais amenas. Então, uma hipótese a ser considerada é se há resposta em produtividade de fibra do algodoeiro à adubação nitrogenada e potássica de cobertura, nessas condições.

O objetivo deste trabalho foi avaliar o efeito de doses de potássio e nitrogênio sobre o estado nutricional, a altura de plantas, os componentes de produção, a produtividade e a qualidade tecnológica da fibra do algodoeiro semeado em segunda safra em sistema adensado.

\section{MATERIAL E MÉTODOS}

O experimento foi conduzido em condições de campo, na área experimental da Fundação Goiás, no Município de Santa Helena de Goiás, Goiás (1750'08'S 50³4'53"'O; altitude $577 \mathrm{~m}$ ), em Latossolo Vermelho (Embrapa, 2006). Antes da instalação, foram coletadas amostras de solo para análise química nas camadas de 0-20 cm e de 20-40 cm de profundidade (Tabela 1).

Os totais diários de precipitação pluvial registrada na área experimental, durante os meses de fevereiro a julho de 2013, foram de 174, 356, 148, 13, 28 e 0 mm (Figura 1), totalizando $718,5 \mathrm{~mm}$.

O algodoeiro foi semeado em 4 de fevereiro de 2013, após a colheita da soja precoce, em sistema de semeadura direta, objetivando população de 150.000 plantas por hectare. O delineamento experimental foi o de blocos ao acaso, com quatro repetições, com os tratamentos distribuídos em esquema fatorial $4 \times 4$, sendo quatro doses de nitrogênio em cobertura $\left(0 \mathrm{~kg} \mathrm{ha}^{-1}, 50 \mathrm{~kg} \mathrm{ha}^{-1}, 100 \mathrm{~kg} \mathrm{ha}^{-1} \mathrm{e}\right.$ $\left.200 \mathrm{~kg} \mathrm{ha}^{-1} \mathrm{de} \mathrm{N}\right)$ e quatro doses de potássio em cobertura ( $0 \mathrm{~kg} \mathrm{ha}^{-1}, 40 \mathrm{~kg} \mathrm{ha}^{-1}, 80 \mathrm{~kg} \mathrm{ha}^{-1}$ e $160 \mathrm{~kg} \mathrm{ha}^{-1} \mathrm{de} \mathrm{K}_{2} \mathrm{O}$ ). Cada parcela foi formada por oito linhas do algodoeiro FM 966 LL, em espaçamento de 0,45 m entre linhas e com 5,0 m de comprimento, sendo as quatro linhas centrais consideradas como área útil. Todas as parcelas receberam $300 \mathrm{~kg}$ $\mathrm{ha}^{-1}$ do adubo 4-30-16, na semeadura. As fontes de $\mathrm{N}$ e de $\mathrm{K}$, na adubação de cobertura, foram ureia e cloreto de potássio. As doses de $\mathrm{N}$ e de $\mathrm{K}$ foram aplicadas simultaneamente no algodoeiro, parceladas em duas aplicações, sendo a primeira adubação aos 25 dias após a emergência (DAE) (V4 - quatro folhas verdadeiras) e a segunda, aos 41 DAE (B1 - primeiro botão floral no primeiro ramo simpodial) (Marur \& Ruano, 2001). 
Os controles de crescimento, de insetos-praga, de plantas daninhas e de doenças fúngicas foram realizados conforme as recomendações de Ferreira (2014), Santos (2015), Sofiatti et al. (2015) e Silva et al. (2017), respectivamente. A desfolha foi feita aos 151 DAE e a colheita foi aos 163 DAE.

No período do florescimento (F8 - primeira flor aberta no oitavo ramo simpodial), as folhas do algodoeiro (quarta folha a partir do ápice da haste principal) foram coletadas em 15 plantas por parcela, acondicionadas em sacos de papel e secadas em estufa de circulação forçada de ar, a $65^{\circ} \mathrm{C}$, por 72 horas, moídas e submetidas à análise dos teores de macro e de micronutrientes (Malavolta et al. 1997).

As variáveis avaliadas foram: teores foliares de macro e de micronutrientes, altura de plantas, número de capulhos por planta, produtividade de algodão em caroço e fibra e composição química das sementes. Amostras de pluma foram tomadas de 20 plantas para análise das características tecnológicas da fibra (comprimento, uniformidade, índice de fibras curtas, resistência, elongação, micronaire ou finura, maturidade, grau de reflexão, grau de amarelo e fiabilidade), por meio do equipamento de High Volume Instrument (HVI). Os resultados obtidos foram analisados estatisticamente por meio da análise de variância (teste F; Pr < 0,01) e da análise de regressão para doses de nitrogênio e potássio. Assumiu-se a significância da interação de doses de nitrogênio e de potássio como critério para eventual desdobramento.

\section{RESULTADOS E DISCUSSÃO}

Os teores foliares de nitrogênio, potássio, magnésio, enxofre, boro e cobre (Tabela 2) apresentam-se abaixo da faixa adequada citada por Yamada et al. (1999), Malavolta (2002) e Kurihara et al. (2013). Os teores propostos por esses autores ainda são utilizados como referência para lavouras de alta produtividade. No entanto, deve-se considerar que eles foram estabelecidos com base em resultados obtidos em condição de potencial genético diferente, em espaçamento e época de cultivos tradicionais, em safra normal. A fase de pleno florescimento, momento em que foi realizada a amostragem foliar, coincidiu com longo período de déficit hídrico (Figura 1), que pode ter influen- ciado negativamente na absorção dos nutrientes, mensurada pela análise foliar (Tabela 2). A correta interpretação de resultados de análises foliares favorece o uso racional de insumos, evita desperdício, melhora o equilíbrio nutricional e, consequentemente, aumenta a produtividade (Serra et al. 2010).

As doses de nitrogênio influenciaram significativamente os teores foliares de nitrogênio, fósforo, magnésio, enxofre e ferro, enquanto as doses de $\mathrm{K}_{2} \mathrm{O}$ alteraram os teores foliares de potássio e magnésio. A interação entre as doses de $\mathrm{N}$ e $\mathrm{K}_{2} \mathrm{O}$ não foi significativa para nenhum nutriente e, portanto, não foi adotado o desdobramento (Tabela 2).

À medida que se aumentou a dose de $\mathrm{N}$, o teor de $\mathrm{N}$ nas folhas do algodoeiro aumentou de forma linear ( $\mathrm{y}=$ $24,1+0,02^{* *} \times R^{2}=0,93$ - Tabela 2), o que também foi verificado por Furlani Júnior et al. (2003). Conforme Breitenbeck \& Boquet (1993), o incremento das doses de $\mathrm{N}\left(0 \mathrm{~kg} \mathrm{ha}^{-1} \mathrm{a}\right.$ $168 \mathrm{~kg} \mathrm{ha}^{-1}$ ) aumentou a absorção e a exportação de N. No entanto, o teor de $\mathrm{N}$ não sofreu influência das doses de $\mathrm{K}_{2} \mathrm{O}$ (Tabela 2). Carvalho et al. (2001), estudando doses de $\mathrm{N}$ e de $\mathrm{K}$ aplicadas em pulverização nas folhas do algodoeiro, encontraram o mesmo comportamento.

$\mathrm{O}$ aumento da dose de $\mathrm{N}$ influenciou positivamente $\mathrm{o}$ teor foliar de $\mathrm{Mg}\left(\mathrm{y}=1,8+0,004^{* * *} \times \mathrm{R}^{2}=0,95\right)$, tendência também observada por Breitenbeck \& Boquet (1993). No entanto, com o acréscimo de $\mathrm{K}_{2} \mathrm{O}$, o teor de $\mathrm{Mg}$ diminuiu de forma quadrática $\left(\mathrm{y}=2,6-0,01^{* *} \mathrm{x}+0,00006^{* *} \mathrm{x}^{2} \mathrm{R}^{2}=\right.$

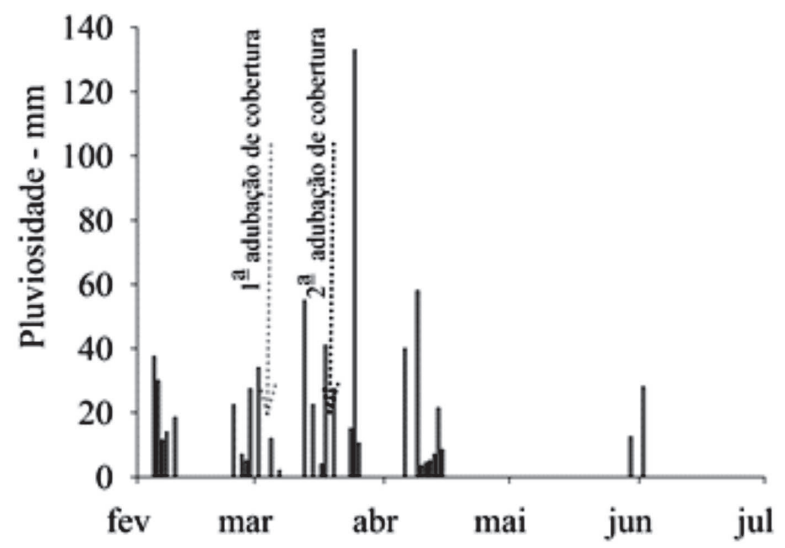

Figura 1: Precipitação diária durante a condução do experimento. Santa Helena de Goiás, GO, 2013.

Tabela 1: Resultados da análise de solo nas camadas de 0-20 cm e de 20-40 cm de profundidade, antes da instalação do experimento

\begin{tabular}{|c|c|c|c|c|c|c|c|c|c|c|}
\hline \multirow{2}{*}{ Prof $^{*}$} & $\mathrm{pH}^{1}$ & $\mathbf{P}^{2}$ & $\mathbf{K}^{3}$ & $\mathrm{Ca}^{4}$ & $\operatorname{Mg}^{5}$ & $\mathrm{H}+\mathrm{Al}^{6}$ & SB $^{7}$ & $\mathrm{CTC}^{8}$ & $\mathbf{V}^{9}$ & \multirow{2}{*}{$\frac{\text { M.O. }{ }^{10}}{(\%)}$} \\
\hline & $\mathrm{CaCl}_{2}$ & $\overline{\left(\mathrm{mg} \mathrm{dm}^{-3}\right)}$ & \multicolumn{7}{|c|}{$\left(\mathrm{mmol}_{\mathrm{c}} \mathrm{dm}^{-3}\right)$} & \\
\hline $0-20$ & 5,3 & 28 & 1,1 & 36 & 14 & 42 & 51,1 & 93,1 & 55 & 3,8 \\
\hline $20-40$ & 5,1 & 28 & 1,2 & 21 & 7 & 52 & 29,2 & 81,2 & 36 & 3,0 \\
\hline
\end{tabular}

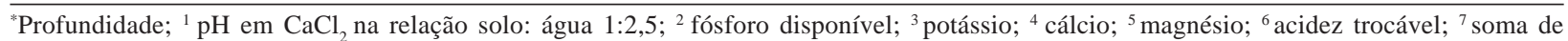
bases $=\mathrm{Ca}+\mathrm{Mg}+\mathrm{K} ;{ }^{8}$ capacidade de troca de cátions $=\mathrm{H}+\mathrm{Al}+\mathrm{SB} ;{ }^{9}$ volume de saturação por bases trocáveis $=\mathrm{SB} / \mathrm{CTC}$ e ${ }^{10}$ matéria orgânica. Métodos: P, K, Ca e Mg - extração pela Resina Trocadora de Íons; M.O. - dicromato/colorimetria (Raij et al. 1986). 
0,84). Esse antagonismo também foi encontrado por Silva et al. (1985). Os teores foliares de enxofre $\left(\mathrm{y}=2,9-0,02^{* *} \mathrm{x}\right.$ $\left.+0,00006^{* *} x^{2} R^{2}=0,92\right)$ e ferro $\left(y=126,2-0,55^{* *} x+0,0018^{*} x^{2}\right.$ $\mathrm{R}^{2}=0,84$ ) foram influenciados pelas doses de $\mathrm{N}$.

Houve baixa absorção de potássio, refletida pelo baixo teor foliar de $\mathrm{K}$, mesmo nas maiores doses de $\mathrm{K}_{2} \mathrm{O}$ (Tabela 2). Este resultado pode estar relacionado com o estresse hídrico ocorrido no período de florescimento, uma vez que o principal mecanismo de transporte no solo do potássio é a difusão, que contribui com 72 a $96 \%$ do total de K absorvido (Oliveira et al. 2004). Além disso, Ferrari et al. (2014) encontraram menor absorção de potássio pelo algodoeiro cultivado em espaçamento de $0,45 \mathrm{~m}$ que em espaçamentos de 0,7 e $0,9 \mathrm{~m}$. As doses crescentes de potássio influenciaram os teores foliares de potássio, os quais apresentaram comportamento quadrático $(\mathrm{y}=12,2$ $\left.+0,05^{* * *} x-0,0003^{*} x^{2} R^{2}=0,97\right)$. O maior teor foliar estimado (14,3 $\left.\mathrm{g} \mathrm{kg}^{-1}\right)$, considerado suficiente por Kurihara et al. (2013), foi obtido com a dose de $83,3 \mathrm{~kg} \mathrm{ha}^{-1}$ de $\mathrm{K}_{2} \mathrm{O}$.

A produtividade de fibra do cultivar FM966LL apresentou comportamento quadrático $\left(\mathrm{y}=1.017+3,015^{\circ} \mathrm{x}-\right.$ $\left.0,014^{\circ} \mathrm{x}^{2} \mathrm{R}^{2}=0,73\right)$, em resposta ao aumento de doses de potássio, sendo a máxima produtividade $\left(1.179,3 \mathrm{~kg} \mathrm{ha}^{-1}\right)$ obtida com a dose de $107 \mathrm{~kg} \mathrm{ha}^{-1}$ de $\mathrm{K}_{2} \mathrm{O}$ (Figura 2). Khalifa et al. (2012) obtiveram aumento da produtividade de algodão em caroço com a fertilização potássica, sendo que a melhor combinação de fertilização foi com $100 \mathrm{~kg} \mathrm{ha}^{-1} \mathrm{de}$ $\mathrm{K}_{2} \mathrm{O}$ e $180 \mathrm{~kg} \mathrm{ha}^{-1}$ de N. Dong et al. (2010) também obtiveram resposta positiva de produtividade às doses de $\mathrm{K}_{2} \mathrm{O}$, em solo com alta e baixa fertilidade. Em trabalho de avaliação de diferentes genótipos quanto à eficiência do uso de potássio, Hassan et al. (2014) observaram diferenças entre genótipos em relação ao aumento de dose, sendo que o genótipo mais eficiente não apresentou diferença de produtividade com doses elevadas do nutriente.

A equação da razão incremental por $\mathrm{kg}$ de $\mathrm{K}_{2} \mathrm{O}$ (dy/dx $=3,015-0,028 x$ ), obtida pela derivada primeira da equação de regressão, apresentou taxas de incremento mais altas para as doses menores, com valores próximos a 3,0 $\mathrm{kg} \mathrm{ha}^{-1}$ de fibra para cada $\mathrm{kg}$ de $\mathrm{K}_{2} \mathrm{O}$ adicionado. Nas doses de 40 e de $80 \mathrm{~kg} \mathrm{ha}^{-1}$, as taxas de incremento estimadas foram de 1,9 e de $0,8 \mathrm{~kg} \mathrm{ha}^{-1}$ de fibra para cada $\mathrm{kg}$ de $\mathrm{K}_{2} \mathrm{O}$, respectivamente. Kappes et al (2015) encontraram incrementos de 3,4 a 5,5 $\mathrm{kg} \mathrm{ha}^{-1}$ de algodão em caroço para cada $\mathrm{kg}$ de $\mathrm{K}_{2} \mathrm{O}$, aplicado em sistema de produção adensado, em segunda safra.

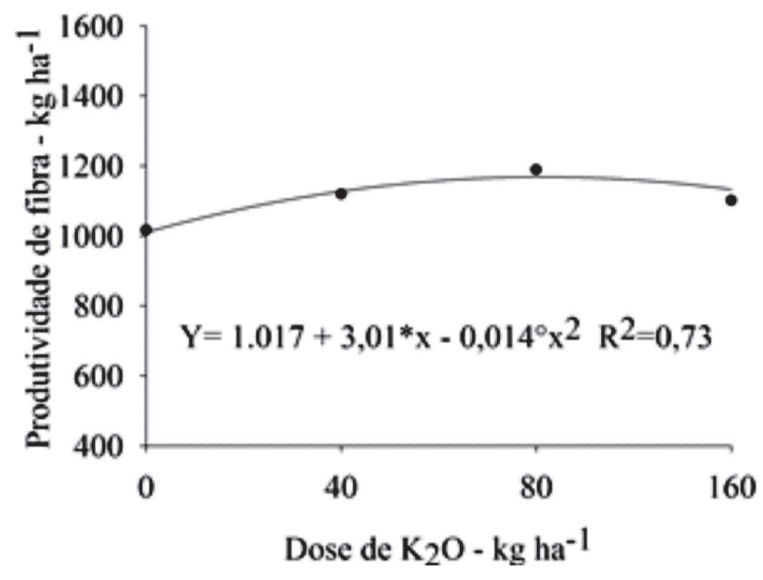

Figura 2: Produtividade de fibra de algodão em função de doses de potássio.

Tabela 2: Teores foliares de macro e de micronutrientes do algodoeiro adensado, safrinha, em função de doses de nitrogênio e de potássio

\begin{tabular}{|c|c|c|c|c|c|c|c|c|c|c|c|}
\hline \multirow{3}{*}{$\frac{\text { Tratamentos }}{\mathrm{N}\left(\mathrm{kg} \mathrm{ha}^{-1}\right)}$} & \multicolumn{11}{|c|}{ Teores foliares de macro e micronutrientes } \\
\hline & $\mathbf{N}$ & $\mathbf{P}$ & $\mathbf{K}$ & $\mathbf{C a}$ & Mg & $\mathbf{S}$ & B & $\mathrm{Cu}$ & $\mathbf{F e}$ & Mn & $\mathbf{Z n}$ \\
\hline & \multicolumn{6}{|c|}{$\left(\mathrm{g} \mathrm{kg}^{-1}\right)$} & \multicolumn{5}{|c|}{$\left(\mathrm{mg} \mathrm{kg}^{-1}\right)$} \\
\hline 0 & 23,8 & 2,8 & 13,2 & 19,1 & 1,7 & 3,0 & 23,4 & 4,0 & 130,0 & 57,9 & 20,4 \\
\hline 50 & 25,0 & 2,3 & 13,8 & 17,5 & 2,1 & 1,9 & 22,1 & 4,2 & 93,0 & 48,9 & 19,3 \\
\hline 100 & 27,0 & 2,3 & 14,6 & 18,8 & 2,3 & 1,9 & 22,4 & 4,1 & 96,9 & 50,7 & 19,6 \\
\hline 200 & 28,1 & 2,4 & 13,5 & 18,3 & 2,6 & 1,8 & 21,9 & 4,1 & 86,8 & 46,8 & 21,1 \\
\hline $\operatorname{Pr}>\mathrm{F}(\mathrm{N})$ & $* *$ & $* *$ & ns & $\mathrm{ns}$ & $* *$ & $* *$ & ns & ns & $* *$ & ns & ns \\
\hline \multicolumn{12}{|l|}{$\mathrm{K}_{2} \mathrm{O}\left(\mathrm{kg} \mathrm{ha}^{-1}\right)$} \\
\hline 0 & 26,4 & 2,6 & 12,2 & 19,0 & 2,6 & 2,2 & 22,2 & 4,1 & 102,2 & 51,9 & 22,5 \\
\hline 40 & 25,4 & 2,4 & 13,7 & 18,4 & 2,0 & 2,1 & 23,0 & 4,0 & 98,8 & 52,0 & 20,7 \\
\hline 80 & 25,6 & 2,5 & 15,1 & 17,6 & 2,1 & 2,2 & 22,2 & 3,9 & 96,7 & 47,6 & 18,6 \\
\hline 160 & 26,4 & 2,4 & 14,1 & 18,7 & 2,0 & 2,1 & 22,4 & 4,2 & 109,0 & 52,9 & 18,6 \\
\hline $\operatorname{Pr}>\mathrm{F}(\mathrm{K})$ & $\mathrm{ns}$ & $\mathrm{ns}$ & $* *$ & ns & $* *$ & ns & $\mathrm{ns}$ & ns & $\mathrm{ns}$ & $\mathrm{ns}$ & $\mathrm{ns}$ \\
\hline $\mathrm{Pr}>\mathrm{F}\left(\mathrm{N}_{\mathrm{x}} \mathrm{K}\right)$ & $\mathrm{ns}$ & $\mathrm{ns}$ & $\mathrm{ns}$ & $\mathrm{ns}$ & $\mathrm{ns}$ & ns & $\mathrm{ns}$ & $\mathrm{ns}$ & $\mathrm{ns}$ & ns & $\mathrm{ns}$ \\
\hline $\mathrm{CV}(\%)$ & 7,7 & 12,3 & 16,3 & 12,1 & 14,3 & 16,0 & 13,7 & 9,3 & 31,2 & 27,0 & 18,7 \\
\hline Média & 26,0 & 2,5 & 13,8 & 18,4 & 2,2 & 2,1 & 22,4 & 4,1 & 101,7 & 51,1 & 20,1 \\
\hline
\end{tabular}

Obs.: ns e **: não significativo e significativo a $1 \%$ de probabilidade pelo teste de $\mathrm{F}$, respectivamente. 
A altura de plantas, o número de capulhos por planta e a produtividade de algodão em caroço e em fibra aumentaram de forma quadrática com o incremento das doses de N. A altura de plantas apresentou comportamento quadrático $\left(y=50,3+0,2^{* *} \mathrm{x}-0,006^{* *} \mathrm{x}^{2} \mathrm{R}^{2}=1,0\right)$ em função das doses de N (Figura 3), mas não sofreu influ- ência das doses de K. Resultados semelhantes da influência de doses de $\mathrm{N}$ sobre a altura de plantas também foram obtidos por Kappes et al (2015), Baraich et al. (2012), Oliveira et al. (2008) e Boquet et al. (2004). No entanto, Furlani Júnior et al. (2003) não constataram esse comportamento.
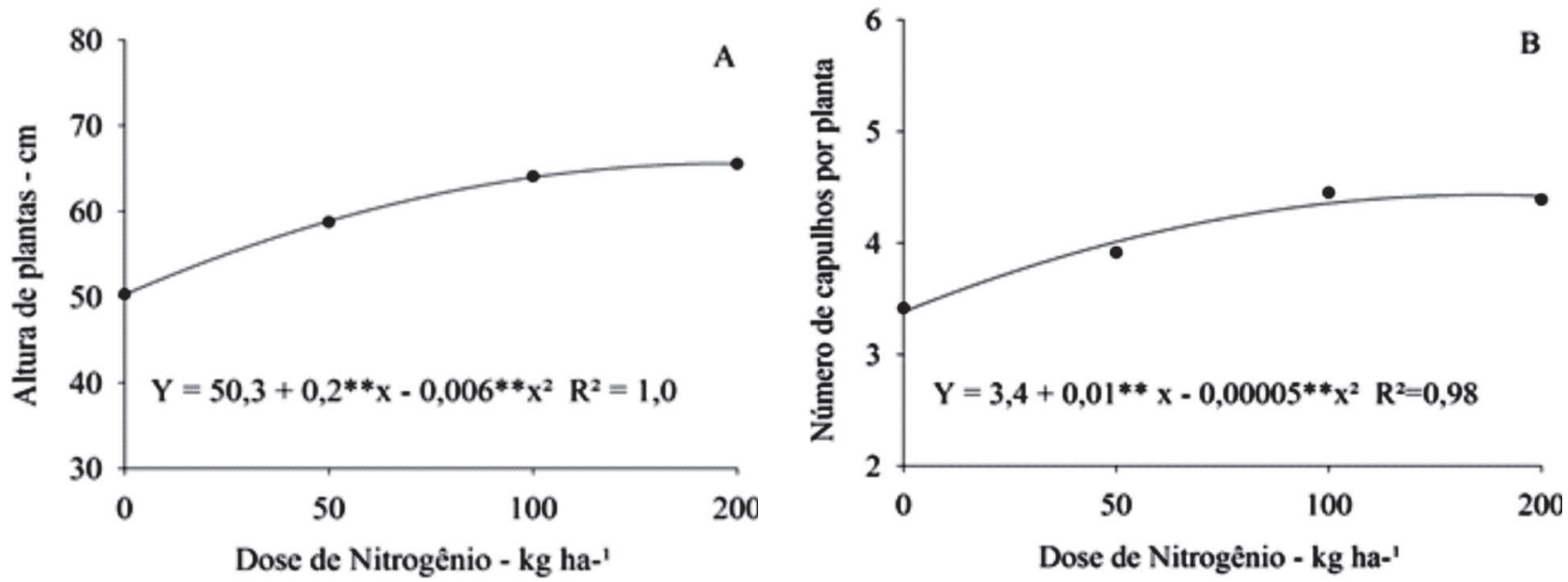

Figura 3: Altura final de plantas (A) e número de capulhos (B) por planta do algodoeiro FM 966LL, em função de doses de nitrogênio.

Tabela 3: Altura de plantas e componentes de produção de algodão, em função de doses de nitrogênio e de potássio em cobertura

\begin{tabular}{|c|c|c|c|c|c|c|}
\hline \multirow{2}{*}{$\frac{\text { Tratamentos }}{\mathbf{N}\left(\mathbf{k g ~ h a}^{-1}\right)}$} & \multirow{2}{*}{$\begin{array}{c}\text { Altura } \\
(\mathrm{cm})\end{array}$} & \multirow{2}{*}{$\begin{array}{c}\text { População } \\
\text { plantas ha }^{-1}\end{array}$} & \multirow{2}{*}{ NCP } & \multirow{2}{*}{$\frac{\text { MC }}{(\mathrm{g})}$} & Prod.algodão & Prod.fibra \\
\hline & & & & & \multicolumn{2}{|c|}{$\left(\mathrm{kg} \mathrm{ha}^{-1}\right)$} \\
\hline 0 & 50,3 & 170.903 & 3,4 & 5,7 & 1.906 & 832 \\
\hline 50 & 58,7 & 163.194 & 3,9 & 6,0 & 2.465 & 1.093 \\
\hline 100 & 64,1 & 159.722 & 4,4 & 5,9 & 2.828 & 1.237 \\
\hline 200 & 65,5 & 159.792 & 4,4 & 6,2 & 2.953 & 1.277 \\
\hline $\mathrm{P}>\mathrm{F}(\mathrm{N})$ & $* *$ & $\circ$ & $* *$ & $* *$ & $* *$ & $* *$ \\
\hline $\mathrm{b} 0(\mathrm{~N})$ & 50,3 & 167.805 & 3,4 & 5,7 & 1.906 & 835 \\
\hline b1 (N) & $0,199^{* *}$ & $-50,3^{*}$ & $0,015^{* *}$ & $0,002^{* *}$ & $13,201^{* * *}$ & $5,944^{* *}$ \\
\hline b2 (N) & $-0,0006^{* *}$ & - & $-0,00005^{* *}$ & - & $-0,0398^{* *}$ & $-0,0187^{* *}$ \\
\hline Desvio (N) & ns & $\mathrm{ns}$ & $\mathrm{ns}$ & $\mathrm{ns}$ & $\mathrm{ns}$ & $\mathrm{ns}$ \\
\hline \multicolumn{7}{|l|}{$\overline{\mathrm{K}_{2} \mathrm{O}\left(\mathrm{kg} \mathrm{ha}^{-1}\right)}$} \\
\hline $0^{2}$ & 59,4 & 159.514 & 4,1 & 6,0 & 2.288 & 997 \\
\hline 40 & 59,9 & 162.917 & 3,9 & 5,9 & 2.660 & 1.167 \\
\hline 80 & 58,9 & 164.722 & 4,1 & 5,9 & 2.581 & 1.129 \\
\hline 160 & 60,4 & 166.458 & 4,1 & 6,0 & 2.624 & 1.146 \\
\hline $\mathrm{P}>\mathrm{F}(\mathrm{K})$ & $\mathrm{ns}$ & ns & ns & $\mathrm{ns}$ & $\circ$ & $\circ$ \\
\hline $\mathrm{b} 0(\mathrm{~K})$ & 59,7 & 159.593 & 4,0 & 6,0 & 2.330 & 1017 \\
\hline b1 (K) & $-0,012^{\mathrm{ns}}$ & $89,457^{\mathrm{ns}}$ & $-0,001^{\mathrm{ns}}$ & $-0,001^{\mathrm{ns}}$ & $6,629^{\mathrm{ns}}$ & $3,015^{\circ}$ \\
\hline b2 (K) & $0,0001^{\mathrm{ns}}$ & $-0,2920^{\mathrm{ns}}$ & $0,00001^{\mathrm{ns}}$ & $9 \times 10^{6 \mathrm{~ns}}$ & $-0,0305^{\mathrm{ns}}$ & $-0,014^{\circ}$ \\
\hline Desvio (K) & $\mathrm{ns}$ & ns & ns & $\mathrm{ns}$ & ns & $\mathrm{ns}$ \\
\hline $\mathrm{P}>\mathrm{F}\left(\mathrm{N}_{\mathrm{x}} \mathrm{K}\right)$ & $\mathrm{ns}$ & $\mathrm{ns}$ & $\mathrm{ns}$ & $\mathrm{ns}$ & $\mathrm{ns}$ & $\mathrm{ns}$ \\
\hline $\mathrm{CV}(\%)$ & 10,2 & 8,1 & 14,1 & 6,6 & 18,0 & 16,9 \\
\hline Média & 59,7 & 163.402 & 4,0 & 5,9 & 2.538 & 1.110 \\
\hline Resíduo & 37,0 & $1,74 \times 10^{8}$ & 0,32 & 0,15 & $2,08 \times 10^{5}$ & $3,5 \times 10^{4}$ \\
\hline
\end{tabular}

NCP - número de capulhos por planta; MC - massa de capulho; Prod. algodão - produtividade de algodão em caroço; Prod. fibra produtividade de fibra. Obs.: ns; $*^{*},{ }^{*},{ }^{\circ}$ : não significativo, significativo a $1 \%$, a $5 \%$ e $10 \%$ de probabilidade pelo teste de $\mathrm{F}$, respectivamente. b0, b1 e b2: estimativa da constante da regressão, estimativa do coeficiente do termo linear da regressão e estimativa do coeficiente do termo quadrático da regressão. Desvio: significância do desvio da regressão. 
A baixa altura de plantas observada no algodão cultivado em segunda safra (Tabela 3) foi devida à restrição hídrica, que se iniciou na segunda quinzena do mês de abril, o que reforça a importância da aplicação da adubação nitrogenada na fase inicial de desenvolvimento vegetativo, no período em que as condições de umidade do solo ainda são adequadas. A parte aérea (caule e folhas) das plantas acumula nutrientes, funcionando como reserva para a redistribuição aos diferentes órgãos da planta no momento de maior demanda para formação das estruturas reprodutivas. Nesta fase, o fruto torna-se o principal dreno de $\mathrm{N}$ da planta (Rosolem \& Mikkelsen 1989), embora, no cultivo em segunda safra, o desenvolvimento reprodutivo e sua alta demanda nutricional coincidam com o término das chuvas.
O número de capulhos por planta aumentou de forma quadrática com o incremento das doses de N (Figura 3), sendo que essa variável relaciona-se positivamente com a produtividade. As máximas produtividades de algodão, em caroço $\left(y=1.905,52+13,2^{* *} x-0,0398^{* *} x^{2} R^{2}=1,0\right)$ e em fibra $\left(y=835+5,9^{* * *} x-0,0187^{* * *} x^{2} R^{2}=0,99\right)$ foram obtidas com as doses estimadas de $165 \mathrm{~kg} \mathrm{ha}^{-1}$ e de $148 \mathrm{~kg} \mathrm{ha}^{-1}$ de $\mathrm{N}$, respectivamente (Figura 4). De acordo com Teixeira et al. (2008), o algodoeiro aumentou a produtividade até a dose de $131 \mathrm{~kg} \mathrm{ha}^{-1}$ de $\mathrm{N}$; mesmo comportamento observado por Boquet et al. (2004), com doses de $\mathrm{N}$ até $118 \mathrm{~kg} \mathrm{ha}^{-1}$.

A equação da razão incremental de $\mathrm{kg}$ de fibra por $\mathrm{kg}$ de $\mathrm{N}(\mathrm{dy} / \mathrm{dx}=5,9-0,0374 \mathrm{x})$, obtida pela derivada primeira da equação de regressão, indicou taxas de incremento
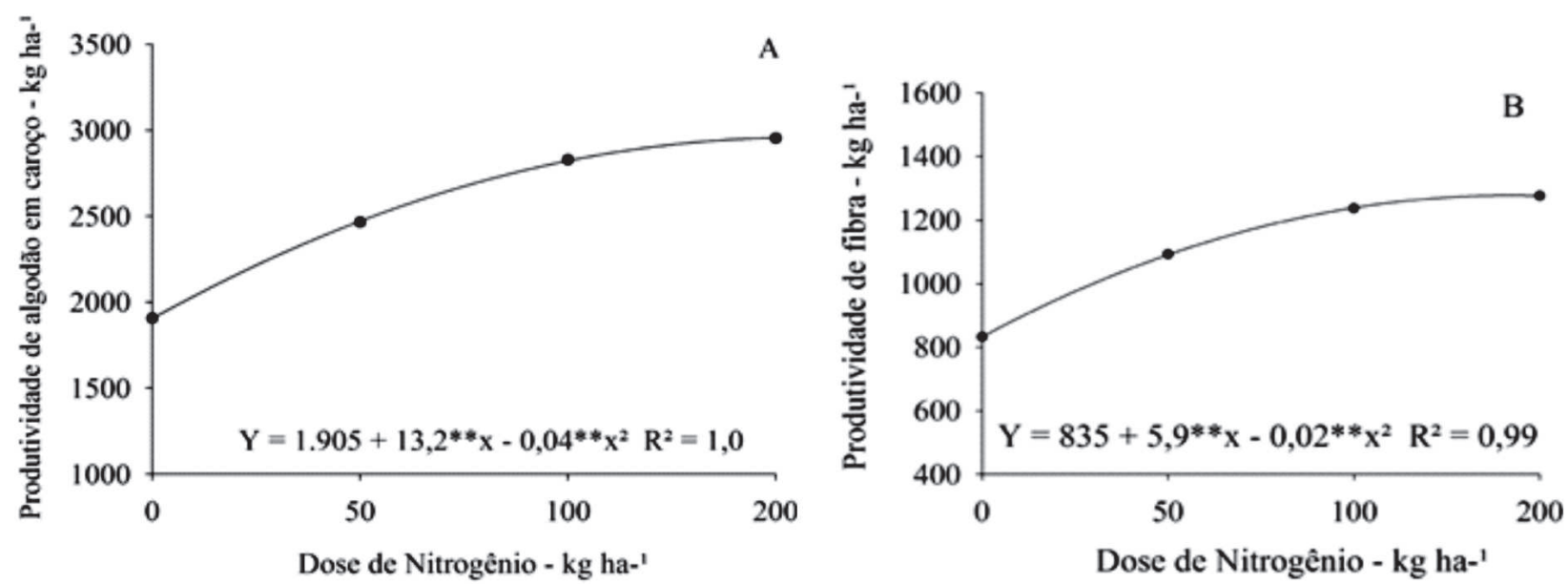

Figura 4: Produtividade de algodão em caroço (A) e fibra (B), em função de doses de nitrogênio.

Tabela 4: Características tecnológicas da fibra: comprimento (UHM), uniformidade (UNF), índice de fibras curtas (SFI), resistência (STR), alongamento (ELG), micronaire (MIC), maturidade (MAT), reflectância (Rd), grau de amarelo (+b) e índice de fiabilidade (CSP) em algodão de segunda safra, em função de doses de $\mathrm{Ne} \mathrm{K}_{2} \mathrm{O}$

Características tecnológicas da fibra

\begin{tabular}{|c|c|c|c|c|c|c|c|c|c|c|}
\hline Tratamentos & UHM & UNF & SFI & STR & $\mathbf{E L}$ & GMIC & MAT & Rd & \multirow[t]{2}{*}{$+\mathbf{b}$} & \multirow[t]{2}{*}{ CSP } \\
\hline $\mathbf{N}(\mathbf{k g ~ h a - 1})$ & $(\mathbf{m m})$ & \multicolumn{2}{|c|}{$(\%)$} & $\left(\right.$ gftex-1) $\left.^{-1}\right)$ & $(\%)$ & $(\mu \mathrm{g}$ pol-1) & \multicolumn{2}{|c|}{$(\%)$} & & \\
\hline 0 & 28,0 & 84,5 & 6,8 & 30,5 & 5,8 & 4,9 & 0,9 & 81,1 & 8,5 & 2.609 \\
\hline 50 & 28,0 & 84,5 & 6,7 & 30,9 & 6,1 & 4,7 & 0,9 & 80,6 & 8,6 & 2.671 \\
\hline 100 & 27,8 & 84,2 & 7,1 & 31,4 & 6,3 & 4,4 & 0,9 & 80,6 & 8,5 & 2.719 \\
\hline 200 & 27,9 & 84,9 & 6,7 & 31,6 & 6,2 & 4,6 & 0,9 & 80,3 & 8,8 & 2.768 \\
\hline $\mathrm{P}>\mathrm{F}(\mathrm{N})$ & $\mathrm{ns}$ & ns & ns & ns & $* *$ & $* *$ & ns & ns & ns & ns \\
\hline \multicolumn{11}{|l|}{$\mathrm{K}_{2} \mathrm{O}\left(\mathrm{kg} \mathrm{ha}^{-1}\right)$} \\
\hline 0 & 27,6 & 84,5 & 6,9 & 31,1 & 6,1 & 4,6 & 0,9 & 81,0 & 8,5 & 2.677 \\
\hline 40 & 28,2 & 84,5 & 6,7 & 31,1 & 6,1 & 4,6 & 0,9 & 81,0 & 8,7 & 2.712 \\
\hline 80 & 28,1 & 84,4 & 6,9 & 30,8 & 6,1 & 4,7 & 0,9 & 80,7 & 8,5 & 2.665 \\
\hline 160 & 27,9 & 84,6 & 6,8 & 31,4 & 6,2 & 4,7 & 0,9 & 80,0 & 8,7 & 2.713 \\
\hline $\mathrm{P}>\mathrm{F}(\mathrm{K})$ & $\mathrm{ns}$ & ns & ns & ns & ns & ns & $\mathrm{ns}$ & ns & ns & ns \\
\hline $\mathrm{P}>\mathrm{F}\left(\mathrm{N}_{\mathrm{x}} \mathrm{K}\right)$ & ns & ns & $\mathrm{ns}$ & $*$ & ns & ns & ns & ns & $\mathrm{ns}$ & ns \\
\hline CV $(\%)$ & 2,7 & 0,9 & 7,0 & 4,8 & 6,0 & 6,4 & 0,8 & 2,7 & 5,9 & 6,1 \\
\hline Média & 27,9 & 84,5 & 6,8 & 31,1 & 6,1 & 4,6 & 0,9 & 80,2 & 8,6 & 2.692 \\
\hline
\end{tabular}

Obs.: ns, * e **: não significativo; significativo a 5 e $1 \%$ de probabilidade pelo teste de $\mathrm{F}$. 
mais altas com as doses menores, com valores próximos a 6,0 $\mathrm{kg} \mathrm{ha}^{-1}$ de fibra para cada $\mathrm{kg}$ de $\mathrm{N}$ adicionado. Nas doses de 50 e $100 \mathrm{~kg} \mathrm{ha}^{-1}$, as taxas de incremento estimadas foram de 4,0 e de 2,0 $\mathrm{kg} \mathrm{ha}^{-1}$ de fibra, para cada $\mathrm{kg}$ de $\mathrm{N}$, respectivamente. Kappes et al (2015) encontraram incremento de $5,7 \mathrm{~kg} \mathrm{ha}^{-1}$ de algodão em caroço para cada kg de N, aplicado em sistema de produção adensado, em segunda safra.

Os componentes de produção e as produtividades de algodão, em caroço e em fibra, não foram influenciados significativamente pela interação dos fatores doses de $\mathrm{N}$ e de $\mathrm{K}_{2} \mathrm{O}$, ou seja, a dose de máxima eficiência de um não foi dependente da dose do outro. Mesmo comportamento foi encontrado por Kappes et al. (2015). Com base nas equações de razão incremental, entre as doses estimadas de 1 a $80 \mathrm{~kg}$, observa-se que o incremento de fibra para cada kg de $\mathrm{N}$ foi mais que o dobro, em comparação com o incremento com cada $\mathrm{kg}$ de $\mathrm{K}_{2} \mathrm{O}$. Portanto, o algodoeiro semeado em segunda safra, em sistema adensado, foi mais responsivo à adubação nitrogenada, sendo as doses de máxima eficiência produtiva, para $\mathrm{N}$, de 148 , e para $\mathrm{K}_{2} \mathrm{O}$, de $107 \mathrm{~kg} \mathrm{ha}^{-1}$, respectivamente.

Em geral, as características tecnológicas da fibra (Tabela 4) apresentaram-se dentro da faixa adequada para a indústria têxtil (Santana et al. 2008; Sestren \& Lima 2011), com exceção para elongação, micronaire e grau de amarelo. A qualidade da fibra sofreu pouca influência do incremento de doses de nitrogênio, exceto para a elongação e o micronaire (Tabela 4). O micronaire $\left(\mathrm{y}=4,9-0,007^{* *} \mathrm{x}+0,00002^{*} \mathrm{x}^{2} \mathrm{R}^{2}=0,91\right)$ e a elongação $\left(y=5,8+0,007^{* *} \mathrm{x}+0,00002^{*} \mathrm{x}^{2} \mathrm{R}^{2}=1,0\right)$ apresentaram comportamento quadrático, sendo que o me- nor valor de micronaire e o maior valor de elongação estimados foram obtidos com a dose de $175 \mathrm{~kg} \mathrm{ha}^{-1} \mathrm{de}$ N. Em algodão adensado, na segunda safra, com semeadura em fevereiro, no momento de deposição das camadas de celulose para a formação da fibra, as possibilidades de temperaturas noturnas abaixo de $20{ }^{\circ} \mathrm{C}$ são altas, podendo-se reduzir a taxa de elongação, (Gipson \& Ray, 1969).

A adubação potássica não interferiu em nenhuma característica tecnológica da fibra. Provavelmente, os teores de $\mathrm{K}$ do solo foram suficientes para não comprometer a fibra, mesmo com sua concentração foliar um pouco abaixo da adequada (Tabela 2). De acordo com Oosterhuis (2001), a deficiência de K interfere negativamente na qualidade da fibra. No entanto, Setatou \& Simonis (1996) afirmam que as características da fibra são principalmente determinadas geneticamente e pouco influenciadas por fatores ambientais.

Os teores de macronutrientes da semente do algodão não foram alterados com o aumento das doses de potássio (Tabela 5), o que indica que não houve consumo de luxo. No entanto, os teores de $\mathrm{N}$ na semente foram influenciados positivamente pelas doses de $\mathrm{N}$, diferentemente do ocorrido com os teores de $\mathrm{P}$ e de $\mathrm{Ca}$, que diminuíram. Hakes \& Grimes (2010) não observaram diferenças nos teores de $\mathrm{P}, \mathrm{K}, \mathrm{S}, \mathrm{Ca}$ e $\mathrm{Mg}$ com o aumento das doses de $\mathrm{N}$ (0 kg ha ${ }^{-1}$ a $\left.134 \mathrm{~kg} \mathrm{ha}^{-1}\right)$.

Os teores de macronutrientes encontrados nas sementes de algodão estão dentro da faixa adequada, conforme Carvalho et al. (2011), exceto para o enxofre e o cálcio, que se mostraram abaixo do nível adequado, sendo ambos imóveis na planta.

Tabela 5: Teores de macronutrientes em caroços de algodão cultivado em safrinha, em função de doses de $\mathrm{N}_{\text {e }} \mathrm{K}_{2} \mathrm{O}$

\begin{tabular}{|c|c|c|c|c|c|c|}
\hline \multirow{2}{*}{$\frac{\text { Tratamentos }}{\text { N }\left(\mathbf{k g ~ h a}^{-1}\right)}$} & \multicolumn{6}{|c|}{ Teores de nutrientes no caroço de algodão } \\
\hline & $\mathbf{N}$ & $\mathbf{P}$ & $\mathbf{K}$ & $\mathbf{C a}$ & Mg & $\mathbf{S}$ \\
\hline & \multicolumn{6}{|c|}{$\left(\mathrm{g} \mathrm{kg}^{-1}\right)$} \\
\hline 0 & 22,9 & 5,4 & 10,4 & 1,4 & 2,4 & 1,2 \\
\hline 50 & 23,7 & 4,8 & 10,3 & 1,3 & 2,6 & 1,2 \\
\hline 100 & 23,7 & 4,5 & 10,2 & 1,2 & 2,4 & 1,2 \\
\hline 200 & 26,5 & 4,5 & 10,2 & 1,2 & 2,4 & 1,3 \\
\hline $\mathrm{P}>\mathrm{F}(\mathrm{N})$ & $*$ & $*$ & ns & $* *$ & ns & ns \\
\hline \multicolumn{7}{|l|}{$\mathrm{K}_{2} \mathrm{O}\left(\mathrm{kg} \mathrm{ha}^{-1}\right)$} \\
\hline 0 & 24,5 & 5,2 & 10,4 & 1,3 & 2,6 & 1,3 \\
\hline 40 & 23,3 & 4,8 & 10,2 & 1,3 & 2,4 & 1,3 \\
\hline 80 & 23,8 & 4,5 & 10,4 & 1,2 & 2,3 & 1,2 \\
\hline 160 & 25,2 & 4,7 & 10,2 & 1,2 & 2,5 & 1,2 \\
\hline $\mathrm{P}>\mathrm{F}(\mathrm{K})$ & $\mathrm{ns}$ & $\mathrm{ns}$ & $\mathrm{ns}$ & $\mathrm{ns}$ & $\mathrm{ns}$ & $\mathrm{ns}$ \\
\hline $\mathrm{P}>\mathrm{F}\left(\mathrm{N}_{\mathrm{x}} \mathrm{K}\right)$ & ns & ns & ns & $\mathrm{ns}$ & ns & ns \\
\hline $\mathrm{CV}(\%)$ & 14,3 & 17,4 & 6,9 & 9,5 & 13,5 & 13,5 \\
\hline Média & 24,2 & 4,8 & 10,3 & 1,3 & 2,5 & 2,5 \\
\hline
\end{tabular}

Obs.: ns, * e ** $^{*}$ não significativo, significativo a 5 e $1 \%$ de probabilidade pelo teste de $\mathrm{F}$. 
Pelos resultados obtidos, observa-se que existem mudanças no estado nutricional do algodoeiro cultivado em segunda safra, período com condições climáticas distintas do período de safra normal, principalmente com relação a menor oferta de água e a temperaturas médias mais baixas. É plausível esperar que as faixas de suficiência dos nutrientes necessitem de adequações e, para tanto, a condução de novos estudos em diferentes ambientes e com os cultivares atuais torna-se necessária, para o estabelecimento de novos padrões, para o algodoeiro cultivado em segunda safra.

\section{CONCLUSÕES}

O algodoeiro cultivado em sistema adensado, na segunda safra, responde, positivamente, em produtividade de fibra, com o aumento das adubações de cobertura nitrogenada e potássica, até as doses de $148 \mathrm{~kg} \mathrm{ha}^{-1}$ e 107 $\mathrm{kg} \mathrm{ha}^{-1}$, respectivamente.

A taxa de incremento de produtividade de fibra por quilo de nitrogênio é o dobro da taxa de incremento por quilo de $\mathrm{K}_{2} \mathrm{O}$.

Os teores de nutrientes das folhas de algodão cultivado em segunda safra são inferiores aos teores considerados suficientes para o algodão cultivado em safra normal.

\section{AGRADECIMENTOS}

Os autores agradecem ao Fundo de Incentivo à Cultura do Algodão em Goiás - Fialgo, pelo apoio financeiro concedido, e à Fundação Goiás, pelo apoio operacional.

\section{REFERÊNCIAS}

Baraich AAK, Baraich AHK, Jamali LA \& Salarzi AU (2012) Effect of nitrogen application rates on growth and yield of cotton varieties. Pakistan Journal of Agriculture - Agricultural Engineering and Veterinary Sciences, 28:115-123.

Breitenbeck GA \& Boquet DJ (1993) Effect of N fertilization on nutrient uptake by cotton. In: Beltwide Cotton Conferences, New Orleans. Proceedings, The National Cotton Council. p.1298-1300.

Boquet DJ, Hutchinson RL \& Breitenbeck A (2004) Long-term tillage, cover crop, and nitrogen rate effects on cotton. Agronomy Journal, 96:1443-1452.

Carvalho MAC, Paulino HB, Furlani-Junior E, Buzetti S, Sá ME \& Athaydes MLF (2001) Uso da adubação foliar nitrogenada e potássica no algodoeiro. Bragantia, 60:239-244.

Carvalho MCS, Ferreira GB \& Staut LA (2011) Nutrição calagem e adubação do algodoeiro. In: Freire EC (Ed.) Algodão no cerrado do Brasil. Aparecida de Goiânia, Associação Brasileira dos Produtores de Algodão. p.677-752.

CONAB (2014) Acompanhamento da safra brasileira de grãos. Disponível em: <http://wwwconabgovbr/OlalaCMS/uploads/arquivos/14_06_10_12_12_37_boletim_graos_junho_2014pdf >. Acessado em: 20 de fevereiro de 2015.
Dong H, Kong X, Li W, Tang W \& Zhang D (2010) Effects of plant density and nitrogen and potassium fertilization on cotton yield and uptake of major nutrients in two fields with varying fertility. Field Crops Research, 119:106-113.

Embrapa - Empresa Brasileira de Pesquisa Agropecuária (2006) Sistema Brasileiro de Classificação de Solos. $2^{\mathrm{a}}$ ed. Rio de Janeiro, Embrapa Solos. 306p.

Ferrari JV, Furlani Junior E, Ferrari S \& Luques APPG (2014) Estado nutricional e produtividade de algodoeiro em função de diferentes espaçamentos e aplicação de regulador de crescimento. Semina: Ciências Agrárias, 35:2285-2296.

Ferreira ACB (2014) Fitorreguladores de crescimento em algodoeiro. Campina Grande, Embrapa Algodão. 4p. (Comunicado técnico, 373).

Furlani Júnior E, Silva NM, Carvalho LH, Bortoletto N, Sabino JC \& Bolonhezi D (2003) Modos de aplicação de regulador vegetal no algodoeiro cultivar IAC-22 em diferentes densidades populacionais e níveis de nitrogênio em cobertura. Bragantia, 62:277-233

Galbieri R, Silva JFV, Asmus GLA, Vaz CMP, Lamas FM, Crestana S, Torres ED, Farias A, Faleiro V de O, Chitarra LG, Rodrigues SMM, Staut LA, Matos ES, Spera ST, Druck S, Magalhães CAS, Oliveira AE, Tachinardi R, Fanan S, Ribeiro NR \& Santos RFS (2014) Áreas de produção de algodão em Mato Grosso: nematoides murcha de fusarium sistemas de cultivo fertilidade e física de solo. Primavera do Leste, Instituto Mato-Grossense do Algodão. 16p. (Circular Técnica, ${ }^{\circ} 8$ ).

Gipson JR \& Ray LL (1969) Fiber elongation rates in five varieties of cotton (Gossypium hirsutum L) as influenced by night temperature. Crop Science Madison, 9:339-341.

Hakes KD \& Grimes DW (2010) Crop water management to optimize growth and yield. In: Stewart JMcD, Oosterhuis D, Heitholt JJ \& Mauney J (Eds.) Physiology of cotton. London, Springer. p.255-264.

Hassan ZU, Arshad M, Basra SMA, Rajpar I, Shah AN \& Galani S (2014) Response of potassium-use-efficient cotton genotypes to soil applied potassium. International Journal of Agriculture \& Biology, 16:771-776.

Hutmacher RB, Travis RL, Rains DW, Vargas RN, Roberts BA, Weir BL, Wright SD, Munk SD, Marsh BH, Keeley MP, Fritsch FB, Munier DJ, Nichols RL \& Delgado R (2004) Response of recent Acala cotton varieties to variable nitrogen rates in the San Joaquim Valley of California. Agronomy Journal, 96:48-62.

Kappes C, Zancanaro L \& Francisco EAB (2015) Nitrogen and Potassium in narrow-row cotton. Revista Brasileira de Ciência do Solo, 40:01-17.

Khalifa K, Al-Chammaa M \& Al-Ain F (2012) Effect of potassium fertilizers on cotton yield and nitrogen uptake efficiency in an Aridisol. Communications in soil Science and plant analysis, $43: 2180-2189$.

Kurihara CH, Venegas VHA, Neves JCL, Novaes RF \& Staut LA (2013) Faixas de suficiência para teores foliares de nutrientes em algodão e em soja, definidas em função de índices DRIS. Revista Ceres, 60:412-419.

Malavolta E (2002) Micronutrientes para algodão e soja. Piracicaba, CENA/USP. 21p.

Malavolta EA, Vitti GC \& Oliveira AS (1997) Avaliação do estado nutricional de plantas: princípios e aplicações. Piracicaba, Potafos. 201p.

Marur CJ \& Ruano O (2001) A reference system for determination of cotton plant development. Revista de Oleaginosas e Fibrosas, 5:243-247. 
Oliveira FA, Silva MNB, Oliveira AP, Santos D, Pereira WE, Oliveira RC \& Gondim SC (2008) Efeito da irrigação e da adubação nitrogenada sobre algumas características de desenvolvimento do algodão colorido verde. Revista Brasileira de Oleaginosas e Fibrosas, 12:49-57.

Oliveira RH, Rosolemca \& Trigueiro RM (2004) Importância do fluxo de massa e difusão no suprimento de potássio ao algodoeiro como variável de água e potássio no solo. Revista Brasileira de Ciência do Solo, 28:39-445.

Oosterhuis D (2001) Physiology and nutrition of high yield in cotton in the USA. Informações Agronômicas, 95:18-24.

Raij B, Quaggio JA \& Silva NM (1986) Extration of phosphorus potassium calcium and magnesium from soils by an ion-exchange resin procedure. Communications in Soil Science and Plant Analysis, 17:547-566.

Rinehardt JM, Edmisten KL, Wells R \& Faircloth JC (2004) Response of ultra-narrow and conventional spaced cotton to variable nitrogen rates. Journal of Plant Nutrition, 27:743755 .

Rochester I, O'halloran J, Maas S, Sands D \& Brotherton E (2007) Monitoring nitrogen use efficiency in your region. Australian Cotton grower, 28:24-27.

Rosolem CA \& Mikkelsen DS (1989) Nitrogen source-sink relationship in cotton. Journal of Plant Nutrition, 12:14171448 .

Rosolem CA, Echer FR, Lisboa IP \& Barbosa TS (2012) Acúmulo de nitrogênio fósforo e potássio pelo algodoeiro sob irrigação cultivado em sistemas convencional e adensado. Revista Brasileira de Ciência do Solo, 36:457-466.

Santana JCF, Wanderley MJR, Beltrão NEM, Azevedo DMP, Leão AB \& Vieira DJ (2008) Características da fibra e do fio do algodão Análise e interpretação dos resultados. In: Beltrão NEM \& Azevedo DMP (Eds.) O agronegócio do algodão no Brasil. Brasília, Embrapa Informação Tecnológica. p.1099-1120.
Santos WJ (2015) Manejo das pragas do algodão, com destaque para o cerrado brasileiro. In: Freire EC (Ed.) Algodão no cerrado do Brasil. Aparecida de Goiânia, Associação Brasileira dos Produtores de Algodão. p.267-364.

Serra AP, Marchetti ME, Vitorino ACT, Novelino JO \& Camacho MA (2010) Desenvolvimento de normas DRIS e CND e avaliação do estado nutricional da cultura do algodoeiro. Revista Brasileira de Ciência do Solo, 34:97-104.

Sestren JA \& Lima JJ (2011) Características e classificação da fibra de algodão. In: Freire EC (Ed.) Algodão no cerrado do Brasil. 2a ed. Aparecida de Goiânia, Associação Brasileira dos Produtores de Algodão. p.891-976.

Setatou HB \& Simonis AD (1996) Effect of time and rate of nitrogen application on cotton. Fertilizer Research, 43:49-53.

Silva JC, Suassuna ND \& Bettiol W (2017) Management of Ramularia leaf spot on cotton using integrated control with genotypes, a fungicide and Trichoderma asperellum. Crop Protection, 94:28-32.

Silva NM, Fuzatto MG, Chiavegato EJ, Ferraz CAM \& Hiroce R (1985) Adubação potássica do algodoeiro: época, modo de aplicação e tipo de fertilizante. Bragantia, 44:263-274.

Sofiatti V, Costa AGF, Ferreira ACB \& Lamas FM (2015) Métodos de controle de plantas daninhas na cultura do algodoeiro. In: Costa AGF \& Sofiatti V (Eds.) Manejo de plantas daninhas na cultura do algodoeiro. Brasilia, Embrapa. p.31-84.

Teixeira IR, Kikuti H \& Borém A (2008) Crescimento e produtividade de algodoeiro submetido a cloreto de mepiquat e doses de nitrogênio. Bragantia, 67:891-897.

Yamada T, Malavolta E, Martins OC, Zancanaro L, Casale H \& Baptista I (1999) Teores foliares de nutrientes observados em áreas de alta produtividade. Piracicaba, Potafós. 25p. 